\title{
ANÁLISIS DE ISÓTOPOS ESTABLES EN COLÁGENO DE HUESOS DE CAMÉLIDOS DE QUEBRADA TULÁN, PUNA DE ATACAMA, PERIODO FORMATIVO TEMPRANO (CA. 3.100-2.400 A.P.)
}

\author{
ANALYSES OF STABLE ISOTOPES IN CAMELIDS COLLAGEN BONES \\ FROM TULÁN RAVINE, ATACAMA PUNA, EARLY FORMATIVE PERIOD \\ (CA. 3, 100-2,400 BP)
}

Patricio López ${ }^{1}$ Isabel Cartajena ${ }^{2}$ y Lautaro Núñez ${ }^{1}$

\begin{abstract}
Se presentan los resultados de los análisis de isótopos estables $\left(\delta^{13} \mathrm{C}\right.$ y $\left.\delta^{15} \mathrm{~N}\right)$ realizados sobre colágeno de huesos de Lama guanicoe (guanaco) y Lama glama (llama) de los sitios Tulán-85 y Tulán-54, asignados al Formativo Temprano (ca. 3.100-2.400 a.p.) y emplazados al sureste de la Puna de Atacama (II Región-Chile). Las muestras zooarqueológicas se seleccionaron mediante criterios anatómicos y morfométricos. Los resultados señalan diferencias en la dietas de ambas especies, explicables por los cambios altitudinales de la vegetación relacionada a la Quebrada Tulán, que se relacionarían con los emplazamientos de caza y pastoreo.

Palabras claves: Puna de Atacama, Quebrada Tulán, camélidos sudamericanos, isótopos estables.
\end{abstract}

This paper presents the results of isotope analysis $\left(\delta^{13} C\right.$ y $\left.\delta^{15} N\right)$ conducted on bone collagen found in Lama guanicoe and Lama glama remains from Tulán-85 and Tulán-54 archaeological sites. Both sites have been dated to the Early Formative Period (ca. 3,100-2,400 BP) and are located southeast of the Atacama Puna basin. Faunal samples were selected using anatomical and morphometric criteria. The results indicate divergences in the diets of both species, reflecting vegetation variation in the Tulán Quebrada caused by altitude differences and linked to hunting and herding areas.

Key words: Atacama Puna basin; Tulán Ravine, South American camelids, stable isotopes.

En recientes investigaciones en arqueología, el uso de isótopos estables ha contribuido a un conocimiento más acabado de la dieta humana y sus cambios a lo largo del tiempo (DeNiro 1985; Falabella et al. 2007; Finucane et al. 2006; Newsome et al. 2004; Tieszen y Chapman 1992; Tykot 2004). Los buenos resultados obtenidos en problemas relacionados con la movilidad de grupos humanos, uso del espacio, la emergencia de cultivos o bien como indicadores de cambios ambientales, han fomentado el uso creciente de este método en otros registros. Es el caso de los restos zooarqueológicos, puesto que reflejan condiciones ambientales específicas, además son excelentes intermediarios para la comprensión de los procesos involucrados en el manejo y transformación humana de un medio a lo largo de una secuencia de tiempo. Si bien el uso de isótopos estables se ha extendido a diversos problemas a lo largo del orbe, su introducción en la arqueología sudamericana es relativamente reciente (Barberena 2002; Barberena et al. 2009; Falabella et al. 2007; Finucane et al. 2006; Gil et al. 2009; Gómez Otero et al. 2000; Guichón et al. 2001; Mengoni Goñalons 2007; Novelillo et al. 2004; Panarello y Fernández 2002; Panarello et al. 2006; Tieszen y Chapman 1992; Slovak et al. 2009; Tykot et al. 2009; Turner et al. 2010). En Chile, los isótopos estables han sido utilizados para el estudio de dieta humana dentro de áreas de investigación específicas, siendo una línea que se encuentra en una fase inicial de desarrollo (Falabella et al. 2007; López 2010; Tieszen y Chapman 1992; Tykot et al. 2009).

El presente trabajo aborda los resultados preliminares obtenidos de los análisis de isótopos estables $\left(\delta^{13} \mathrm{C}\right.$ y $\left.\delta^{15} \mathrm{~N}\right)$ en restos de camélidos (Lama guanicoe y Lama glama) de los sitios Tulán-85 y

\footnotetext{
1 Instituto de Investigaciones Arqueológicas y Museo, Universidad Católica del Norte, Le Paige 380, San Pedro de Atacama, Chile. patriciolopezmend@yahoo.es; lautaro.nunez@hotmail.com

2 Facultad de Ciencias Sociales, Departamento de Antropología, Universidad de Chile, Ignacio Carrera Pinto 1045, Ñuñoa, Santiago, Chile.icartaje@uchile.cl
} 
Tulán-54 (TU-85 y TU-54 en adelante), asignados ambos al Formativo Temprano de la Puna de Atacama (II Región, Chile. Ver Figura 1). El interés en la aplicación de este método se basa en la exploración de indicadores que permitan profundizar en el uso del espacio y manejo de los camélidos por parte de los grupos humanos asentados en la Quebrada Tulán hacia los ca. 3.100 a 2.400 años a.p. Las evidencias arqueofaunísticas y contextuales para el área de estudio hacia el Formativo Temprano señalan el manejo de rebaños de camélidos junto a la caza tanto de guanacos como vicuñas (Cartajena et al. 2007, 2009; Núñez et al. 2006). Esta convergencia económica entre caza y crianza de camélidos debió implicar mecanismos de control y distribución de pasturas y forrajes de las tierras altas y bajas de la Puna de Atacama, en función de los requerimientos de animales domésticos y la obtención de recursos mediante la caza. A través del análisis isotópico en restos de camélidos domésticos y silvestres se busca caracterizar la dieta de estas especies, con el fin de explorar si las diferencias observables pueden atribuirse a la explotación de diversos cotos de caza y áreas de pastoreo. Los resultados son preliminares y de tipo exploratorio, no obstante, constituyen una aproximación al estudio isotópico de restos zooarqueológicos en la zona.

\section{Ambientes, Ocupación Humana, Camélidos e Isótopos Estables}

Las zonas de Prepuna y Puna de Atacama se localizan entre las latitudes $22^{\circ}$ y $24^{\circ} \mathrm{S}$, en la II Región de Antofagasta, Provincia del Loa (Norte Árido de Chile). La Prepuna, que abarca el margen superior de la zona desértica absoluta y los paisajes altoandinos, se caracteriza por una orografía dominada por quebradas que posibilitan la conexión entre las tierras bajas con la Puna. La sequedad en esta zona es casi absoluta, con precipitaciones estivales producto del Invierno Altiplánico o Invierno Boliviano. La Puna de Atacama o Puna Salada, por su parte, se caracteriza por una meseta cuyo relieve alcanza una altura promedio de $4.000 \mathrm{msm}$ con una serie de volcanes que alcanzan los 5.000 a $6.000 \mathrm{msm}$ (Núñez y Santoro 1988).

El ambiente en Quebrada Tulán se caracteriza en la actualidad por un régimen árido, con lluvias estivales que varían entre los pisos altitudinales, puesto que para la cota del Salar la lluvia alcanza

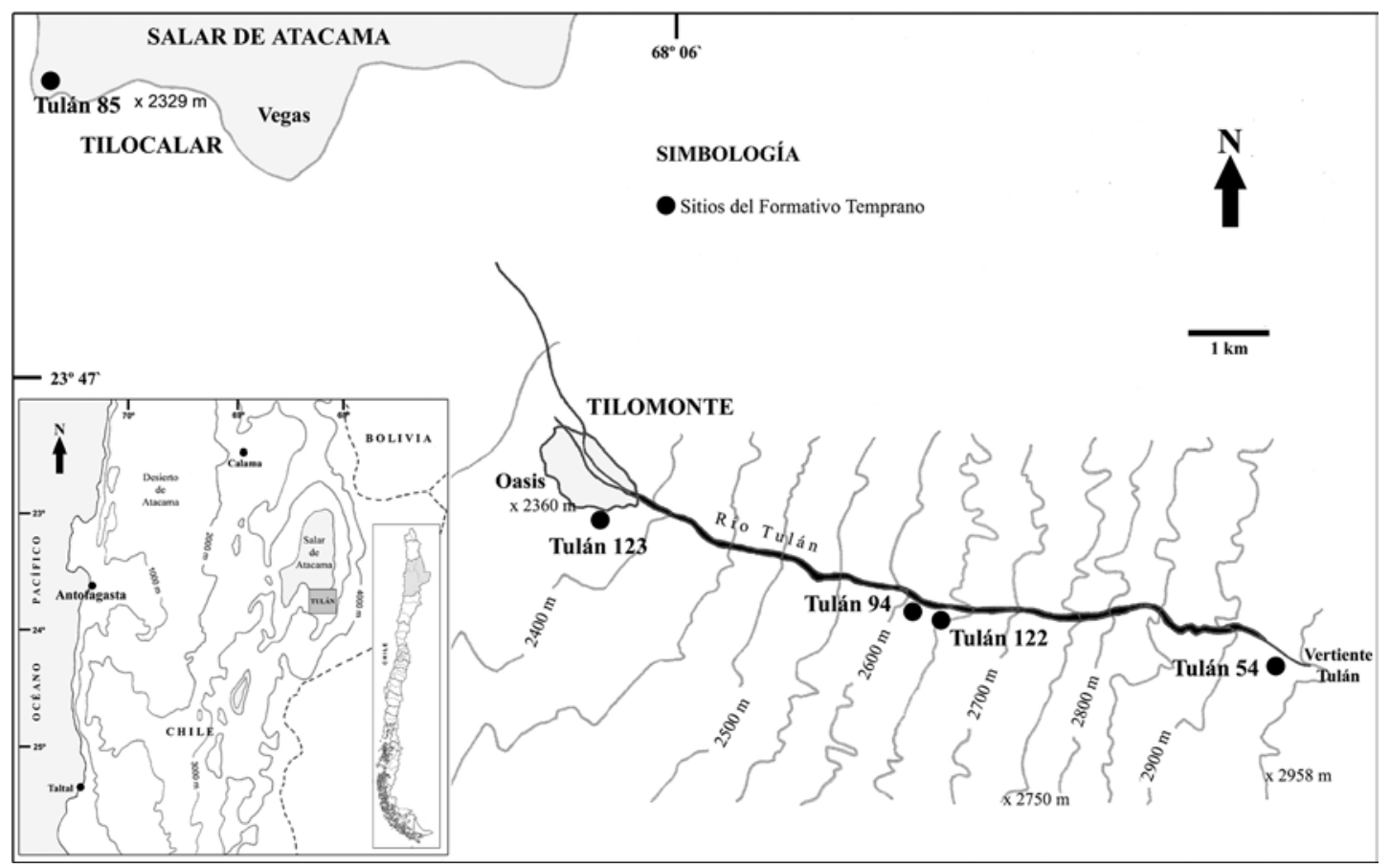

Figura 1. Ubicación de los sitios asignados al Formativo Temprano en la Quebrada Tulán (Figura tomada y modificada de Núñez et al. 2009).

Location of sites assigned to the Early Formative Period in the Tulán ravine (Figure taken and modified from Núñez et al. 2009). 
los $10 \mathrm{~mm}$ al año, mientras que para la alta Puna alcanza los $200 \mathrm{~mm}$ (Núñez et al. 2009). De acuerdo a esto, las cotas de altura son las que condicionan la disponibilidad de recursos y convergencia de distintas especies animales, reconociéndose los siguientes pisos ecológicos: (1) Piso Tilocalar (2.300 a $2.380 \mathrm{msm}$ ), ubicado en el borde oriental del Salar de Atacama y cuyos recursos alcanzan su óptimo en octubre, noviembre y diciembre, aunque con una vega que se mantiene estable todo el año. Dentro de este piso se encuentra emplazado el sitio TU-85, en un ambiente ideal para la cría de camélidos; (2) Piso Tilomonte (2.380 a $2.750 \mathrm{msm}$ ), emplazado en el extremo inferior del área de desagüe del río Tulán y cercano al oasis de Tilomonte; (3) Piso Tulán (2.750 a 3.500 msm), que engloba parte de la quebrada homónima y quebradas secas que llegan hasta la alta Puna. Incluye además coberturas vegetacionales del Tolar que crecen por encima de los $3.000 \mathrm{msm}$ concentrando la mayor cantidad de forraje con una marcada estacionalidad (80\% del total de las especies). El sitio Tulán-54 fue registrado en este piso ecológico; y (4) Piso Miñiques (3.500 a $5.650 \mathrm{msm}$ ), en donde se observa la mayor concentración de fauna andina en la cabecera de las lagunas de Miscanti y Miñiques (Núñez et al. 2009). Estos pisos complementarios se encuentran articulados en el área de estudio a través de la Quebrada Tulán que se extiende entre las vegas del Salar de Atacama y las tierras altas (Núñez et al. 2006)

En esta zona convergieron una serie de condiciones socioculturales y ambientales favorables que posibilitaron la transición del Arcaico Tardío a las sociedades del Formativo Temprano. El surgimiento del Formativo, asociado a la emergencia de prácticas pastoralistas, se da en el piso de quebradas intermedias como Tulán, entre la alta puna y los oasis piemontanos (Núñez et al. 2006). Estos ambientes funcionaron como refugios para los grupos humanos durante el Holoceno Medio debido al estrés ambiental provocado por condiciones más áridas que las actuales, caracterizado en las tierras altas por lagos poco profundos y de características hipersalinas. Entre los 3.600 y 3.000 a.p., se observa un incremento de la humedad con la recuperación de los niveles lacustres hasta alcanzar las condiciones modernas, coincidente con las ocupaciones formativas (Grosjean et al.1997; Núñez et al. 1997). A través de los análisis polínicos fue posible observar estas condiciones de mayor humedad, sin embargo, también eventos de aridez hacia fines del Formativo Temprano. Si bien se identificaron especies vegetales actualmente presentes en los diferentes pisos vegetacionales (González et al. 2011), es necesario considerar que dependiendo de las condiciones de humedad estas especies pudieron haber variado en su distribución altitudinal. Estas variaciones no debieron tener el impacto de aquellas observadas durante el Holoceno Temprano donde la pluviosidad triplicó los rangos actuales y la vegetación altoandina descendió hasta los pisos más bajos (Betancourt et al. 2000; Grosjean et al. 1997).

La Quebrada Tulán presenta condiciones orográficas caracterizadas por un arroyo de aproximadamente $30 \mathrm{~km}$ que desemboca hacia el Salar de Atacama, cubriendo un espacio con condiciones favorables como pasaje de interacción. Las condiciones de la Quebrada Tulán se asocian directamente con los hábitats naturales de camélidos silvestres, lo que estimuló la caza de estos animales; no obstante, estos hábitats fueron usados también para la crianza. Tanto la caza como el mantenimiento de rebaños presentan una serie de preguntas relacionadas con la movilidad de los grupos humanos inmersos dentro de esta economía de apropiación y producción. En el caso de las evidencias de caza cabe preguntarse por los cotos utilizados por los grupos del Formativo Temprano de Quebrada Tulán, considerando la posición intermedia de este ambiente con relación a los oasis piedemontanos y la alta puna, además de la estacionalidad como una variable relevante en la abundancia de camélidos. Por lo anteriormente expuesto, se considera que el uso de isótopos estables en restos de camélidos tanto silvestres como domésticos tiene gran potencial para discutir diferentes aspectos relacionados con la explotación y manejo de estos animales. En la presente investigación, el análisis isotópico busca generar expectativas relacionadas al uso del espacio durante el Formativo Temprano en Quebrada Tulán, considerando los ambientes asociados a este sector, dentro de una exploración inicial que incorpora muestras tanto de guanacos como de llamas.

\section{Materiales y Métodos}

Las muestras seleccionadas para el análisis isotópico provienen de los sitios TU-54 y TU-85 (Figura 1), sitios trabajados por Núñez et al. (2006). TU-54 corresponde a un asentamiento emplazado en el borde sur de la Quebrada Tulán. Hasta ahora 
se han identificado varios sectores que muestran una compleja organización del espacio, destacando un gran templete central y algunas estructuras de carácter habitacional a su alrededor datados entre los 2.380 a 3.080 a.p. Bajo las capas de un montículo estratificado, se registró una estructura ovalada compuesta por un muro perimetral construido con grandes bloques verticales y con nichos rectangulares a nivel del piso. En este piso se registraron las inhumaciones de 24 neonatos humanos, los que fueron dispuestos en pozos apegados al muro perimetral y también al interior de la estructura central (Núñez et al. 2006). Por su parte, el sitio Tulán-85 se localiza en el borde sureste de la cuenca del Salar de Atacama, con un patrón artefactual y dataciones de 3.140 a 2.360 a.p. cal correspondiente a la fase Tilocalar (Formativo Temprano). Este yacimiento se caracteriza por un área de descarte de forma monticular, compuesta por depósitos estratificados que alcanzan gran profundidad. En el sector meridional se registraron estructuras circulares y subcirculares aglomeradas, construidas con grandes lajas verticales con un patrón propio de la fase Tilocalar (Núñez et al. 2006).

Los especímenes analizados corresponden a ocho primeras falanges de animales adultos (mayores de 12-18 meses como edad mínima), las que fueron seleccionadas de un conjunto mayor de 27 especímenes. La selección de primeras falanges obedece al potencial que tienen estas unidades anatómicas para discriminar grupos de tamaños (Cartajena et al. 2007). Para controlar las diferencias de tamaño, se separaron las falanges anteriores de las posteriores debido a que difieren en longitud, sobre todo en el grupo de camélidos grandes (Cartajena 2002, 2009). La separación de este conjunto en muestras de Lama guanicoe y L. glama se basó en criterios osteométricos para el Formativo Temprano en donde al considerar en forma conjunta las medidas ancho de la superficie articular proximal (BFp sensu von den Driesch 1999) y profundidad de la epífisis proximal (Dp sensu von den Driesch 1999) se observan dos grupos. Un grupo se ubica en el rango inferior de dispersión (afín a L. guanicoe) y el otro en el rango superior de tamaño (afín a L. glama) (Cartajena 2002, 2009; Cartajena et al. 2007). La aplicación del test $t$ de Student para la medida BFp y para la medida Dp de las ocho muestras seleccionadas de ambos sitios posibilitó rechazar la hipótesis nula de que ambas medidas son iguales al 95\% de confianza $(t=-6,69$; $p<0,05$ y $t=-6,45 ; p<0,05$, respectivamente). Estos datos implican que hay una separación clara entre ambos grupos de tamaño para ambas medidas, en donde cuatro especímenes corresponderían a Lama glama y otras cuatro falanges a Lama guanicoe.

Los análisis de isótopos estables fueron realizados sobre colágeno de hueso en el Center for Applied Isotope Studies (CAIS) de la University of Georgia con un espectrómetro de masas modelo Finnigan MAT252. Para controlar los valores isotópicos se midió la concentración de $\mathrm{C}(\mathrm{C} \%)$ y $\mathrm{N}(\mathrm{N} \%)$ y se calculó la razón $\mathrm{C} / \mathrm{N}$ para identificar la preservación del colágeno. Para esto, se compara la razón de $\mathrm{C} / \mathrm{N}$ con los rangos aceptables (2,9 a 3,6) discutidos en la literatura referida al tema (De Niro 1985). Los valores de $\delta^{13} \mathrm{C}\left({ }^{13} \mathrm{C} /{ }^{12} \mathrm{C}\right)$ se expresan en partes por mil (\%o) siguiendo al estándar internacional PeeDee Belemnite (PDB) con un error menor al 0,1\%o y las del $\delta^{15} \mathrm{~N}\left({ }^{15} \mathrm{~N} /{ }^{14} \mathrm{~N}\right)$ en partes por mil (\%o) según el estándar internacional para el nitrógeno atmosférico (Air) con un error menor de 0,2\%o.

\section{Valores de $\delta^{13} \mathrm{C}$ y $\delta^{15} \mathrm{~N}$ en los camélidos de Quebrada Tulán}

Los valores de $\delta^{13} \mathrm{C}$ y $\delta^{15} \mathrm{~N}(\mathrm{C} / \mathrm{N})$ obtenidos a partir de las primeras falanges de camélidos de los sitios TU-54 y TU-85 caen en su mayoría dentro de valores cercanos al rango de lo aceptable (De Niro 1985). Las muestras TU-85/14, TU-85/2 y TU-85/8 son las únicas que están dentro de este rango, mientras que el resto de los valores van desde los $\mathrm{C} / \mathrm{N}=$ 2,6\% a 2,9\%o (Tabla 1). Las muestras de TU-54, en su totalidad presentaron valores por debajo del rango ideal. La o las causas que inciden en estos valores, especialmente en el sitio TU-54, no han sido identificadas y se encuentran en proceso de estudio.

En TU-54, los valores de $\delta^{13} \mathrm{C}$ obtenidos van de $\operatorname{los}-12,6 \%$ a $-16,4 \%$ (Tabla 1 ). Las firmas isotópicas expresadas por grupos de tamaños comprenden para el conjunto de Lama glama valores de $-13,9 \pm, 02 \%$ o (TU54/6) y $-15,4 \pm, 04 \%$ o (TU54/9), mientras que para $L$. guanicoe los valores obtenidos son de $-12,6 \pm, 35 \%$ o (TU54/7) y de $-16,4 \pm, 73 \%$ o (TU54/14).

En el caso de TU-85, los valores de $\delta^{13} \mathrm{C}_{\mathrm{Col}}$ obtenidos van de los $-13,9 \%$ a $-16,8 \%$ o (Tabla 1 ). Si se comparan las firmas isotópicas de acuerdo a los grupos de tamaños, el conformado por L. glama tiene valores de $-13,9 \pm, 01 \%$ o (TU85/8) y $-16,4 \pm, 07 \%$ o (TU85/14), mientras que para el grupo de $L$. guanicoe los valores obtenidos son de 
Tabla 1. Resultados de $\delta^{13} \mathrm{C}$ y $\delta^{15} \mathrm{~N}$ a partir de las muestras de TU-54 y TU-85. $\delta^{13} \mathrm{C}$ and $\delta^{15} \mathrm{~N}$ results from $\mathrm{TU}-54$ and $T U-85$ samples.

\begin{tabular}{llrrrrr}
\hline Muestra & Grupo de tamaño & $\delta 13 \mathrm{C} \pm 1 \sigma$ & $\delta 15 \mathrm{~N} \pm 1 \sigma$ & $\% \mathrm{C}$ & $\% \mathrm{~N}$ & $\mathrm{C} / \mathrm{N}$ \\
\hline TU54/6 & Grande/Llama & $-13,9 \pm .02$ & $13,2 \pm, 37$ & 39,5 & 14,1 & 2,8 \\
TU54/9 & Grande/Llama & $-15,4 \pm .04$ & $13,3 \pm, 14$ & 42,8 & 16,3 & 2,6 \\
TU54/7 & Grande/Guanaco & $-12,6 \pm .35$ & $12,3 \pm, 18$ & 44,2 & 16,3 & 2,7 \\
TU54/14 & Grande/Guanaco & $-16,4 \pm .73$ & $8,6 \pm, 01$ & 40,3 & 14 & 2,9 \\
TU85/8 & Grande/Llama & $-13,9 \pm .01$ & $10,9 \pm, 32$ & 41,5 & 13,4 & 3,1 \\
TU85/14 & Grande/Llama & $-16,4 \pm .07$ & $9,8 \pm, 88$ & 32,9 & 11,2 & 2,9 \\
TU85/2 & Grande/Guanaco & $-14,1 \pm .08$ & $11 \pm, 21$ & 42,2 & 14,5 & 2,9 \\
TU85/7 & Grande/Guanaco & $-16,8 \pm .35$ & $7 \pm, 01$ & 40,2 & 14,1 & 2,9 \\
\hline
\end{tabular}

$-14,1 \pm, 08 \%$ o (TU85/2) y de $-16,8 \pm, 4 \%$ o (TU85/7). Los valores de $\delta^{15} \mathrm{~N}$ presentan diferencias entre las muestras de L. guanicoe en comparación a las de L. glama, siendo en el caso de las llamas los valores en promedio más altos, especialmente las muestras provenientes de TU-54 (Figura 2).

A partir de los valores isotópicos dentro de los grupos de tamaño escogidos se observa para el caso del conjunto compuesto por L. guanicoe una mayor dispersión de los valores de $\delta^{13} \mathrm{C}$ con un mínimo de $-16,5 \%$ y un máximo de $-12,6 \%$ (Figura 2). Para las falanges asignadas a $L$. glama los valores de $\delta^{13} \mathrm{C}$ presentan un mínimo de $-16,41 \%$ y un máximo de $-13,9 \%$. Estos valores indican un rango mayor en los valores de L. guanicoe en comparación a los

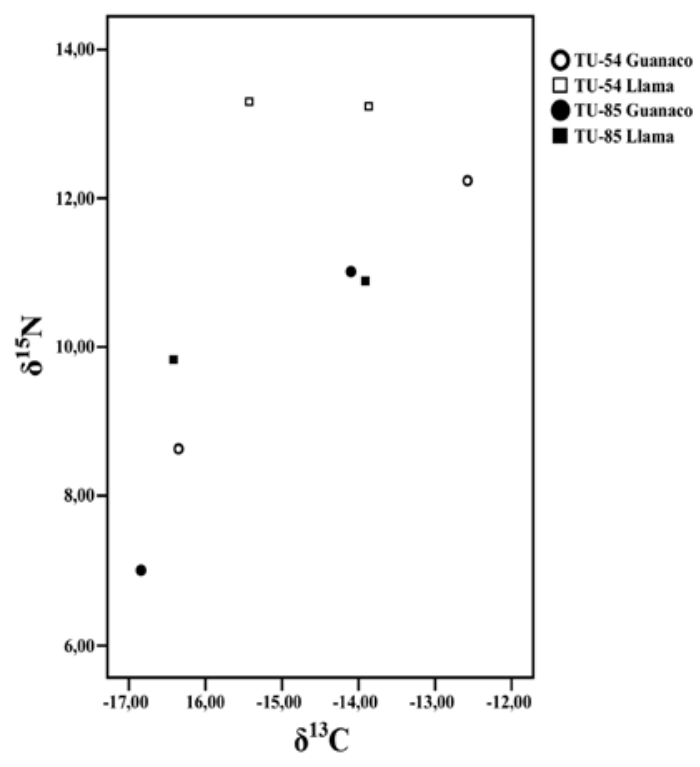

Figura 2. Gráfico de dispersión con los valores de $\delta^{13} \mathrm{C}_{\mathrm{Col}} \mathrm{y}$ $\delta^{15} \mathrm{~N}$ por sitio y afinidad taxonómica.

Scatterplot of $\delta^{13} C_{C o l}$ and $\delta^{15} \mathrm{~N}$ values by site and taxonomic affinity. extremos de L. glama (Figura 2). En el caso de los valores de $\delta^{15} \mathrm{~N}$, la media para $L$. glama es mayor en comparación a $L$. guanicoe, en donde el valor mínimo de $\delta^{15} \mathrm{~N}$ para el primer grupo corresponde a 9,8\%o, mientras que el máximo es de 13,3\% . Para el caso de $L$. guanicoe los valores son menores a los de L. glama con un mínimo de 6,9\%o y un máximo de 12,3\%o.

\section{Relaciones de los valores isotópicos de los sitios TU-54 y TU-85 con la cobertura vegetacional de la Puna de Atacama}

La composición de la vegetación está fuertemente influenciada por la diferencia altitudinal, lo que incide en diversas formaciones vegetales o pisos de vegetación. En la zona de estudio se pueden observar al menos tres formaciones vegetales: matorral desértico, matorral bajo desértico y matorral bajo de altitud (González et al. 2011; Luebert y Pliscoff 2006). No obstante, para este trabajo se consideraron las zonas vegetacionales propuestas por Quade et al. (2007) puesto que se conocen los ciclos fotosintéticos para muchas de las especies vegetales que las componen y además, las divisiones coinciden con los pisos ecológicos propuestos por Núñez y Santoro (1988) para el área de Tulán. Estas grandes zonas vegetacionales asociadas al Salar de Atacama corresponden a la Prepuna (2.500 a $3.200 \mathrm{msm}$ ), Tolar o Puna (3.200 a $4.000 \mathrm{msm}$ ), Estepa Andina (4.000 a $4.500 \mathrm{msm}$ ) y la zona Subnival (>4.500 msm) (Quade et al. 2007). En su mayoría las plantas distribuidas en estas zonas poseen ciclos fotosintéticos $\mathrm{C}_{3}$, salvo algunas de tipo CAM y $\mathrm{C}_{4}$ (Quade et al. 2007). En la Prepuna dominan plantas como Cistanthe salsoides $\left(\mathrm{C}_{3}\right)$, Gilia crassifolia $\left(\mathrm{C}_{3}\right)$, Acantholippia deserticota $\left(\mathrm{C}_{3}\right)$, Ephedra breana $\left(\mathrm{C}_{3}\right)$ y Atriplex 
imbricada $\left(\mathrm{C}_{4}\right)$. En la zona de Tolar se observan en la parte baja especies como Fabiana denudata $\left(\mathrm{C}_{3}\right)$, Baccharis tola y Baccharis boliviensis $\left(\mathrm{C}_{3}\right)$, mientras que en la parte alta (3.700 a $4.000 \mathrm{msm}$ ) dominan taxa como Parastrephia quadrangularis $\left(\mathrm{C}_{3}\right)$ y Parastrephia lepidophylla $\left(\mathrm{C}_{3}\right)$. A diferencia de los matorrales perennes $\mathrm{C}_{3}$, los pastos anuales en el Tolar son $\mathrm{C}_{4}$, como Aristida adscensionis, Bouteloua simplex, Enneapogon devauxii y Munroa decumbens (Quade et al. 2007). La Estepa Andina se encuentra dominada por una variedad de pastos como Festuca chrysophylla $\left(\mathrm{C}_{3}\right)$, Deyeuxia spp. y Stipa frigida $\left(\mathrm{C}_{3}\right)$. Por último, en la zona subnival destacan plantas de cojín del género Azorella y Pycnophyllum (Quade et al. 2007).

$\mathrm{Si}$ se consideran los valores isotópicos medios disponibles para las zonas aledañas a la Quebrada Tulán se observan valores más bajos a medida que se asciende por sobre los $3.000 \mathrm{msm}$. Los valores para las plantas $\mathrm{C}_{3}, \mathrm{C}_{4}$ y CAM no se solapan entre distancias definidas cada $500 \mathrm{~m}$ de altitud, con una diferencia de $-0,2 \%$ para el caso de las $\mathrm{C}_{3}\left(\right.$ Media $_{\mathrm{C} 3 / 3300-}$ $3500 \mathrm{msm}=-22,4 \%$; Media $_{\mathrm{C} 3 / 3500-4000 \mathrm{msm}}=-22,6 \%$ ) $)$, de $-0,04 \%$ o para las $\mathrm{C}_{4}\left(\right.$ Media $_{\mathrm{C} 4 / 3300-3500 \mathrm{msm}}=-14,9 \%$; Media $_{\mathrm{C} 4 / 3500-4000 \mathrm{msm}}=-14,9 \%$ ) y de $0 \%$ o $\left(\right.$ Media $_{\mathrm{CAM} / 3300-3500 \mathrm{msm}}=-14,9 \%$; Media $_{\mathrm{CAM} / 3500-}$ $4000 \mathrm{msm}=-14,9 \%$ ). Bajo los $1.500 \mathrm{msm}$ esta relación es inversa con una diferencia de $-1,8$ para el caso de las $\mathrm{C}_{3}\left(\right.$ Media $_{\mathrm{C} 3 / 1500-1000 \mathrm{msm}}=-22 \%$; Media $_{\mathrm{C} 3 / 1000-0 \mathrm{msm}}=-23,8 \%$ o), de $-3,3$ para las $\mathrm{C}_{4}$ Media $_{\mathrm{C} 4 / 1500-1000 \mathrm{msm}}=-18,4 \%$; Media $_{\mathrm{C} 4 / 1000}$ $0 \mathrm{msm}=-15,1 \%$ ) $\mathrm{y}$ con plantas de ciclo CAM únicamente presentes entre los 0 y $500 \mathrm{msm}$ $\left(\right.$ Media $_{\mathrm{CAM} / 1000-0 \mathrm{msm}}=-12,6 \%$ o). En el caso de las $\mathrm{C}_{3}$, los valores son en promedio más altos a la media global que es ca. -27\%o (Quade et al. 2007). Al respecto, estudios realizados por Ehleringer et al. (1998) señalan que las tasas de isótopos de carbono son infrecuentemente altas en plantas $\mathrm{C}_{3}$ en los ambientes costeros del Desierto de Atacama. Esta situación se debe a las bajas concentraciones de $\mathrm{CO}_{2}$ intercelular como una respuesta adaptativa a las condiciones del medio. Por esto, los valores de $\delta^{13} \mathrm{C}$ y $\delta^{15} \mathrm{~N}$ obtenidos en restos faunísticos o humanos en la zona deben considerar estas variaciones antes de realizar cualquier comparación con áreas aledañas.

\section{Discusión}

En la Tabla 2 se sintetizan los promedios de los valores de $\delta^{13} \mathrm{C}$ de los grupos botánicos presentes en el área de estudio. Dentro de estos grupos los de mayor abundancia según Quade et al. (2007) corresponden a especies de Poaceae (gramíneas),

Tabla 2. Valores promedio de $\delta^{13} \mathrm{C}$ de las plantas $\mathrm{C}_{3}, \mathrm{C}_{4}$ y CAM de las zonas aledañas a la Quebrada Tulán (Datos tomados y modificados de Quade et al. 2007). Average $\delta^{13} C$ values of $C_{3}, C_{4}$ and CAM plants from the vicinity of the Tulán Ravine (Data taken and modified from Quade et al. 2007).

\begin{tabular}{lcrr}
\hline \multicolumn{1}{c}{ Taxa } & Ciclo & Media $\delta 13 \mathrm{C}$ & Altitud (msm) \\
\hline Familia Poaceae & $\mathrm{C}_{3}$ & $-23,7$ & $3.100-4.200$ \\
Familia Fabaceae & $\mathrm{C}_{3}$ & $-22,4$ & $3.475-4.070$ \\
Familia Solanaceae & $\mathrm{C}_{3}$ & $-22,8$ & $425-4000$ \\
Familia Asteraceae & $\mathrm{C}_{3}$ & $-22,6$ & $429-4.700$ \\
Familia Malvaceae & $\mathrm{C}_{3}$ & $-23,3$ & $130-3.571$ \\
Familia Boraginaceae & $\mathrm{C}_{3}$ & $-23,3$ & $3.200-4.000$ \\
Familia Verbenaceae & $\mathrm{C}_{3}$ & $-20,4$ & 3.745 \\
Familia Amaranthaceae & $\mathrm{C}_{4}$ & $-15,1$ & $429 / 3.942$ \\
Chenopodium aff. pinnatum & $\mathrm{C}_{3}$ & $-24,3$ & 429 \\
Familia Ephedraceae & $\mathrm{C}_{3}$ & $-22,1$ & $2.600-3789$ \\
Familia Polemoniaceae & $\mathrm{C}_{3}$ & $-23,3$ & $3.744-3.305$ \\
Familia Cactaceae & $\mathrm{CAM}_{3}$ & $-12,9$ & $130-3475$ \\
Echinopsis deserti & $\mathrm{C}_{3}$ & $-23,5$ & $429-425$ \\
Familia Portulacaceae & $\mathrm{C}_{3}$ & $-22,1$ & $3.356-3.123$ \\
Cistanthe sp. & $\mathrm{C}_{4}$ & $-18,4$ & $1.460-1.240$ \\
Familia Loasaceae & $\mathrm{C}_{3}$ & $-22,4$ & $1.460-1.240$ \\
Familia Aizoaceae & $\mathrm{C}_{3}$ & $-21,9$ & 975 \\
Mesembryanthemum crystallinum & $\mathrm{CAM}_{3}$ & $-13,9$ & 425 \\
Familia Euphorbiaceae & $\mathrm{C}_{3}$ & $-22,6$ & $429-130$ \\
Familia Brassiacoae & $\mathrm{C}_{3}$ & $-25,6$ & $3.200-429$ \\
Familia Frankeniaceae & $\mathrm{C}_{3}$ & $-22,1$ & 425 \\
\hline
\end{tabular}


Asteraceae, Amaranthaceae, Fabaceae, Solanaceae, Loasaceae, Cactaceae, Malvaceae, Portulacaceae y Euphorbiaceae. A estos grupos se suma la introducción en baja proporción de Zea mays (maíz) durante el Formativo Temprano en Quebrada Tulán, especie que se agrupa dentro de las plantas $\mathrm{C}_{4}$ (Núñez et al. 2009).

$\mathrm{Si}$ se consideran los datos relacionados a las preferencias alimentarias de los camélidos domésticos y silvestres para zonas desérticas y semidesérticas de altura se observa una clara preferencia en el consumo de gramíneas tanto en sectores de pajonales como de bofedales. Los estudios realizados por Cortés et al. (2003) en los sectores cordilleranos de Chile norte-centro sobre la dieta del guanaco muestran una alta inclusión de Stipa chrysophylla (Poaceae) tanto en los periodos secos como húmedos. En el caso de los hábitos alimentarios del guanaco en la costa del Desierto de Atacama, Raedeke y Simonetti (1988) identificaron una mayor concentración de líquenes como base de la dieta durante todas las estaciones, seguido por Euphorbia lactiflua (Euphorbiaceae) y Solana sp. (Solanaceae). En el caso de la llama, los estudios realizados por Castellaro et al. (2004) en las praderas altiplánicas de la Provincia de Parinacota (XV Región de Chile) identificaron una alta concentración de gramíneas (i.e. Festuca nardifolia y Agrostis tolucensis) y Juncaceae (Oxychloe andina) en las zonas de bofedal y pajonal, además de Asteraceae (Parastrephia lucida) en el Tolar.

Los valores de los especímenes TU54/6 y TU54/7 indican un consumo tanto de plantas $\mathrm{C}_{3} \mathrm{y}$ $\mathrm{C}_{4}$, mientras que las piezas TU54/9 y TU54/14 sus valores sugieren una ingesta prácticamente exclusiva de plantas $\mathrm{C}_{3}$. Este sitio se emplaza a una cota de $2.952 \mathrm{msm}$ en un área en la que el Tolar hace su aparición por sobre los $3.000 \mathrm{~m}$ de altitud con un gran potencial para el forraje. En el sitio TU-85 los valores de los especímenes TU85/2 y TU85/8 apuntan a un consumo tanto de plantas $\mathrm{C}_{3}$ y $\mathrm{C}_{4} \mathrm{y}$ en el caso de las falanges TU85/7 y TU85/14 los valores indican un consumo mayoritario de $\mathrm{C}_{3}$ (Figura 3). Si bien, estos antecedentes sugieren un panorama muy similar al del sitio TU-54, los valores que señalan un consumo de $\mathrm{C}_{3} \mathrm{y}_{4}$ tienden a ser negativos, por tanto con una mayor incidencia de plantas $\mathrm{C}_{3}$. Este sitio se emplaza en el borde del Salar de Atacama a $2.318 \mathrm{msm}$ donde los recursos alcanzan en los meses de octubre, noviembre y diciembre su óptimo con una vega estable durante todo el año (Núñez et al. 2009).
En cuanto a los valores separados a través de grupos de tamaño y afinidad taxonómica, se observa para el caso de Lama glama valores isotópicos que indican un consumo mayoritario de plantas $\mathrm{C}_{3}$ (TU54/9 y TU85/14) en dos especímenes, mientras que el resto del conjunto presenta firmas isotópicas más positivas producto del consumo de $\mathrm{C}_{3} \mathrm{y}_{4}$ (TU85/8 y TU54/7). Una situación similar se observa en las muestras de L. guanicoe con dos especímenes cuyos valores indican un consumo dominado por plantas $\mathrm{C}_{3}$ (TU54/14 y TU85/7), mientras que dos muestras agrupan valores propios de plantas tanto $\mathrm{C}_{3}$ como $\mathrm{C}_{4}$ (TU54/7 y TU85/2). La pieza (TU54/7) presenta un valor muy positivo de $-12,6 \%$ (Dieta isotópica $=-17,7$ ) lo que se debe a un consumo elevado de plantas $\mathrm{C}_{4}$. A partir del promedio de las plantas $\mathrm{C}_{3}$ y $_{4}$ de alto potencial dietético ${ }^{1}$ para guanacos y llamas en pisos altitudinales que van de los ca. 2.500 a 4.500 msm comparadas con los valores isotópicos de cada muestra zooarqueológica, se observa que los modelos que mejor definen las muestras que incorporan $\mathrm{C}_{3}$ y $\mathrm{C}_{4}$ en su dieta involucran un consumo cercano a un $50 \%$ de cada conjunto o bien con predominancia de $\mathrm{C}_{3}$. La excepción corresponde al espécimen TU54/7 (L. guanicoe) con un consumo dominado por plantas de tipo $\mathrm{C}_{4}$ que bordeó el $70 \%$ de su dieta.

En los especímenes óseos con un dominio de plantas de tipo $\mathrm{C}_{3}$ se observa en el caso de la muestra de guanaco TU-85/7 un porcentaje cercano al $90 \%$ de ingesta de este conjunto, mientras que en el espécimen de guanaco TU-54/14 el valor de $\delta^{13} \mathrm{C}$ comprende un ca. $80 \%$ de plantas $\mathrm{C}_{3}$. En el caso de las muestras de llama, la falange TU-85/14 alcanza el valor más alto con un ca. 80 a $90 \%$ de plantas $\mathrm{C}_{3}$ en su dieta seguido por la muestra de TU-85/9 con un ca. $70 \%$. Por esto y en base a toda a la información precedente en cuanto a la distribución altitudinal de las plantas desde la Prepuna hasta la Estepa Andina y a los valores de $\delta^{13} \mathrm{C}$ de las falanges de ambos sitios, es posible señalar que todas las muestras que incorporaron $\mathrm{C}_{4}$ en sus dietas se alimentaron en zonas cuyas altitudes van de los ca. 2.900 a $4.000 \mathrm{msm}$

Los restos arqueofaunísticos del sitio TU-54 provienen mayoritariamente de una estructura ceremonial donde se registró el consumo de camélidos adultos y subadultos (destacando la alta presencia de neonatos en el templete), cuyos restos se depositaron en los estratos de basura. Los análisis osteométricos indican la presencia tanto de guanaco 


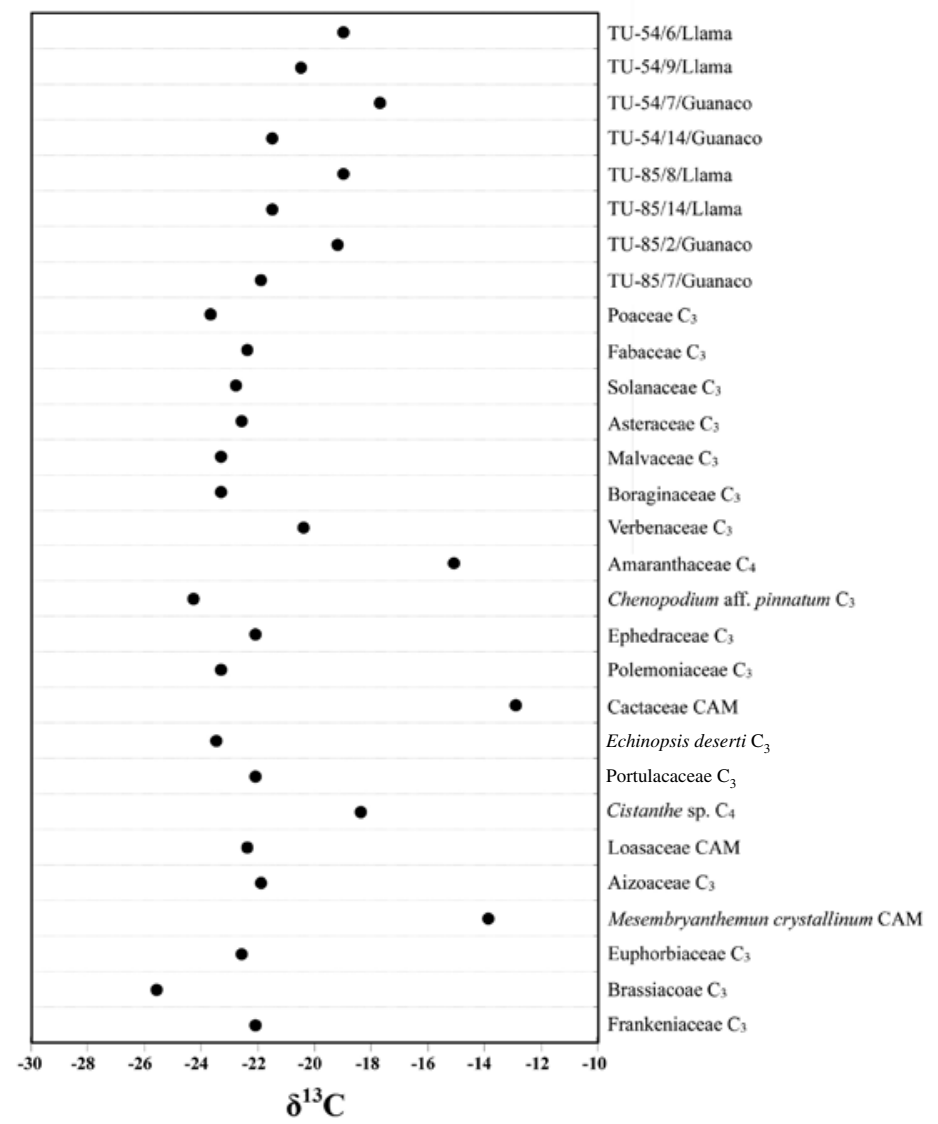

Figura 3. Comparación entre los valores de $\delta^{13} \mathrm{C}_{\mathrm{Col}}$ de las muestras de los sitios TU-54 y TU-85 con las muestras de $\delta^{13} \mathrm{C}_{\mathrm{Col}}$ de las plantas del área de estudio (valores tomados de Quade et al. 2007). Los valores de camélidos de los sitios TU-54 y TU-85 son expresados con el factor $+5,1 \%$ o (ver Tykot et al. 2009).

Comparison between $\delta^{13} C_{C o l}$ values from the TU-54 and TU-85 samples and plants from the study area (values taken from Quade et al. 2007). For TU-54 and TU-85 values a fractionation factor of $+5,1 \%$ o was applied (see Tykot et al. 2009).

y llama, mientras que en el grupo pequeño destaca la vicuña (Cartajena 2009; Núñez et al. 2006). Este sitio se emplaza en las cercanías del Tolar por lo que el área circundante posee recursos para el consumo humano y forrajeo animal. Por esto, cabe preguntarse si las muestras del sitio TU-54 analizadas en la presente investigación sugieren una relación espacial entre este emplazamiento arqueológico y las áreas de captura y crianza de camélidos. Al respecto, los valores isotópicos que predominan en las muestras de L. guanicoe y L. glama que tienden a un consumo tanto de plantas $\mathrm{C}_{3}-\mathrm{C}_{4}$ presentan valores más positivos a los del sitio TU-85. Estos valores indican una ingesta mayor de plantas $\mathrm{C}_{4}$ lo que se relaciona directamente al emplazamiento del sitio, puesto que en la zona del Tolar y a partir de los $3.000 \mathrm{msm}$ la frecuencia de coberturas vegetacionales con ciclos $\mathrm{C}_{4}$ es mayor durante todo el año y para algunas especies durante el verano. Para el caso de las muestras con firmas isotópicas exclusivamente $\mathrm{C}_{3}$, las que corresponden a un guanaco y a una posible llama, sus valores se explican mediante un escenario en el que se hayan incorporados camélidos cazados y criados de zonas más bajas.

Las muestras zooarqueológicas de TU-85 provienen del interior de estructuras, dentro de un área cubierta por una extensa ocupación de depósitos de basura y de un montículo de desechos sin estructuras (Núñez et al. 2006). Los análisis de polen realizados en TU-85 en los niveles asociados a la ocupación de Formativo Temprano señalan altos valores de Cyperaceae probablemente asociados a un cuerpo de agua cercano, además de una vegetación similar 
al actual piso altitudinal intermedio, con altos valores de Ephedra y Poaceae tipo Stipa (González et al. 2011). Hacia el final de la secuencia polínica se observan condiciones de mayor aridez con ausencia de Cyperaceae y presencia de taxa de los pisos desérticos (González et al. 2011). Dentro de estos antecedentes no se observan a nivel polínico restos de Amaranthaceae, grupo que congrega especies de tipo $\mathrm{C}_{4}$. Pese a esto, los datos de Núñez et al. (2009) señalan la presencia de macrorrestros de Atriplex sp. en el sitio TU-85 durante la Fase Tilocalar, género del cual algunas de sus especies son utilizadas en la actualidad como forraje para camélidos y ganado introducido (Villagrán et al. 2003). Esta evidencia plantea la posibilidad de que plantas que se hayan desarrollado en zonas más altas fueran trasladadas y utilizadas por los ocupantes de TU-85 como combustible, material de construcción y posiblemente como complemento alimentario para los camélidos. Sobre lo mismo, los valores de ambos especímenes de L. glama de TU-85 indican un consumo mayoritario de plantas $\mathrm{C}_{3}$ para una de las muestras (TU85/14) lo que se condice con esta inclusión marginal de taxa con ciclo $\mathrm{C}_{4}$, mientras que el espécimen TU85/8 posee valores similares de $\mathrm{C}_{3}$ y $\mathrm{C}_{4}$. Algo similar ocurre con las dos muestras de guanacos, con valores para una de éstas en la que predominan las $\mathrm{C}_{3}$ (TU85/7) y la otra con proporciones casi iguales de $\mathrm{C}_{3}$ y $\mathrm{C}_{4}$. También es interesante la presencia de macrorrestros de Amaranthaceae puesto que son ricas en $\delta^{13} \mathrm{C}$ y $\delta^{15} \mathrm{~N}$ (Tieszen y Chapman 1992 ; Turner et al. 2010). En el caso de datos con valores altos de $\delta^{15} \mathrm{~N}$ se asocian valores también elevados de $\delta^{13} \mathrm{C}$, mientras que las muestras que presentan valores $\delta^{13} \mathrm{C}$ empobrecidos también poseen valores bajos de $\delta^{15} \mathrm{~N}$. Lo anterior podría relacionarse con el uso de plantas de este grupo como forraje sobre todo para animales domésticos.

En el caso de los valores de $\delta^{15} \mathrm{~N}$, como se mencionó anteriormente, tienden a ser más bajos en Lama guanicoe en comparación a Lama glama, y más altos en el sitio TU-54 con relación a TU-85. La edad de los individuos es uno de los factores que suele afectar las variaciones de $\delta^{15} \mathrm{~N}$; sin embargo, las muestras analizadas de ambos sitios corresponden a animales adultos (Hedges y Reynard 2007). En el caso del tipo de plantas consumidas la ausencia de datos de $\delta^{15} \mathrm{~N}$ para las cubiertas vegetacionales de la zona no permite discutir las diferencias observadas en los camélidos de TU-54 y TU-85. En el caso de los sitios los valores de $\delta^{15} \mathrm{~N}$ de TU-54 son más elevados en comparación al sitio TU-85, lo que podría explicarse por diferencias en los tipos de suelo o aguas. En el caso de TU-54 se ha constatado además, la presencia de guano al interior de la estructura lo que podría incidir en los valores de $\delta^{15} \mathrm{~N}$ (Tykot et al. 2009). Independiente de esto, los valores de $\delta^{15} \mathrm{~N}$ de ambos sitios, especialmente TU-54, son muy altos en comparación a otros resultados obtenidos en los Andes centrales y meridionales (Mengoni Goñalons 2007: Tabla 1; Tieszen y Chapman 1992: Tabla 6; Turner et al. 2010: Tabla 2), por lo que es necesario investigar sus causas.

\section{Conclusiones}

La metodología, análisis y resultados expuestos en el presente trabajo son preliminares y de tipo exploratorios. No obstante, es posible sugerir para el caso de las muestras de L. glama dos probables escenarios para las áreas de pastura. Un primer escenario apunta a dos zonas delimitadas por sus diferencias altitudinales. La primera corresponde a una zona ubicada en los pisos altitudinales comprendidos entre los ca. 2.900 a $4.000 \mathrm{msm}$ con un énfasis en el Tolar (Piso Tulán) y asociado directamente a sitios como TU-54, TU-55 y TU-122, y una segunda zona localizada en las cercanías de las actuales vegas de Tilocalar cercana a TU-85. La presencia de dos zonas de pasturas concuerda con la información etnográfica del área, donde es posible visualizar familias y estancias ubicadas tanto en las vegas de Tilocalar como en Quebrada Tulán, no obstante, ambos grupos utilizaban estacionalmente los pisos altoandinos (Núñez 2000). Si bien para los dos contextos arqueológicos estudiados las muestras reflejan ambas zonas de pastura, los antecedentes contextuales sugieren actividades comunales que pueden haber integrado a distintos grupos de la Fase Tilocalar distribuidos a lo largo de la Quebrada Tulán. Sin embargo, a este respecto, la evidencia isotópica no permite identificar trazas en el caso de la llama de un pastoreo estacional en tierras altas debido a la presencia anual y durante el verano de plantas con ciclos $\mathrm{C}_{4}$ dentro de coberturas en donde se distribuyen también $\mathrm{C}_{3}$. Finalmente, es necesario considerar que los valores de $\delta^{13} \mathrm{C}$ reflejan la dieta promedio del individuo y por tanto enmascara las posibles variaciones estacionales (Yacobaccio et al. 2009).

Un segundo posible escenario es la inclusión de plantas $\mathrm{C}_{4}$ en la dieta de algunos hatos de camélidos 
de manera independiente a los pisos altitudinales. Un ejemplo para este escenario es la incorporación del maíz en la dieta de llamas y alpacas lo que ha sido documentado etnoarqueológicamente y observado a partir de datos arqueológicos concretos (ver Mengoni Goñalons 2007). De acuerdo a los datos disponibles para la Quebrada Tulán la presencia de Zea mays tanto en TU-54 como en TU-85 es de baja proporción y se vincula principalmente a prácticas rituales más que al consumo en términos de subsistencia (Núñez et al. 2009). En los casos en que se han observado valores más positivos en camélidos y por ende interpretados por un aumento de plantas $\mathrm{C}_{4}$ como el maíz, el $\delta^{13} \mathrm{C}$ tiende a ser más positivo, mientras que los valores de $\delta^{15} \mathrm{~N}$ son más bajos en comparación a los valores obtenidos en los sitios de Tulán (Mengoni Goñalons 2007). Asi mismo, los coeficientes de variación de los valores tanto de los guanacos y llamas de ambos sitios presentan una correlación positiva y significativa $(r=0.93079, P<0.001)$ lo que indica el posible uso de pasturas similares. Todos estos antecedentes sugieren por tanto que este segundo escenario es el menos plausible.

Consideramos que el uso de isótopos estables presenta un gran potencial para el estudio de la domesticación y caza de camélidos en la Puna de Atacama. $\mathrm{Al}$ respecto, los tres puntos abordados referentes a las dietas de guanacos y llamas, uso del espacio y movilidad estacional poseen distintos grados de visibilidad a partir de los rastros isotópicos. A futuro se debe abordar este análisis con muestras y contextos que den cuenta de toda la secuencia ocupacional del área de estudio para así identificar e interpretar la mayor cantidad de variables involucradas dentro de esta relación entre ambiente y ocupaciones humanas.

Agradecimientos: Este trabajo fue realizado en el marco del Proyecto FONDECYT 1070040. Nuestros agradecimientos a los tres evaluadores anónimos por sus comentarios y sugerencias, los que ayudaron a mejorar el manuscrito. Asimismo, deseamos agradecer a Fernanda Falabella por la ayuda brindada.

\section{Referencias Citadas}

Barberena, R. 2002. Los límites del mar. Isótopos estables en la Patagonia Meridional. Sociedad Argentina de Antropología, Buenos Aires.

Barberena, R., A. Zangrando, A. Gil, G. Martínez, G. Politis, L. A. Borrero y G. Neme 2009. Guanaco (Lama guanicoe) isotopic ecology in southern South America: spatial and temporal tendencies, and archaeological implications. Journal of Archaeological Science 36:2666-2675.

Betancourt, J. L., C. Latorre, J. A. Rech, J. Quade. y K. A. Rylander 2000. A 22,000-year record of monsoonal precipitation from Northern Chile's Atacama desert. Science 289 (5484):1542-1546.

Cartajena, I. 2002. Los Conjuntos Faunísticos del Arcaico Temprano en la Puna de Atacama, Norte de Chile. Inaugural-Dissertation zur Erlangung des Doktogrades im Fachbereich Geschicths-un kulturwissenschaften der Freien Universität Berlin, Berlín.

- - - 2009. Explorando la variabilidad morfométrica del conjunto de camélidos pequeños durante el Arcaico tardío y el Formativo temprano en Quebrada Tulán, norte de Chile. Revista del Museo de Antropología 2:199-212.

Cartajena, I., L. Núñez y M. Grosjean 2007. Camelid domestication in the western slope of the Puna de Atacama, Northern Chile. Anthropozoologica 42:155-173.

Cartajena, I., M.A. Benavente, L. Núñez y C. Thomas 2009. La utilización de los camélidos durante el Formativo Temprano: Una comparación entre la cuenca del Loa Medio y el Salar de Atacama. En Zooarqueología y Tafonomía en el Confín del Mundo, editado por P. López, I. Cartajena, Ch. García y F. Mena, pp. 181-198. Ediciones Universidad Internacional SEK-Chile, Santiago.
Castellaro, G., T. Ullrich, B. Wackwitz y A. Raggi 2004. Composición botánica de la dieta de alpacas (Lama pacos L.) y llamas (Lama glama L.) en dos estaciones del año, en praderas altiplánicas de un sector de la Provincia de Parinacota, Chile. Agricultura Técnica 64:353-364.

Cortés, A., E. Miranda, J. Rau y J. Jiménez 2003. Feeding habits of guanacos Lama guanicoe in the high Andes of north-central Chile. Acta Theriologica 48:229-237.

De Niro, M. 1985. Postmortem preservation and alteration of in vivo bone collagen isotope ratios in relation to paleodietary reconstruction. Nature 317:806-809.

Ehleringer, J., P. Rundel, B. Palma y H. Mooney 1998. Carbon isotope ratios of Atacama Desert plants reflect hyperaridity of region in northern Chile. Revista Chilena de Historia Natural 71:79-86.

Falabella, F., M. Planella, E. Aspillaga, L. Sanhueza y R. Tykot 2007. Dieta en sociedades alfareras de Chile Central: aporte de análisis de isótopos estables. Chungara Revista de Antropología Chilena 39:5-27.

Finucane, B., P. Maita Agurto y W. Isbell 2006. Human and animal diet at Conchopata, Peru: stable isotope evidence for maize agriculture and animal management practices during the Middle Hrizon. Journal of Archaeological Science 33:1766-1776.

Gil, A., G. Neme, R. Tykot, P. Novellino, V. Cortegoso y V. Durán 2009. Stable isotopes and maize consumption in central western Argentina. International Journal of Osteoarchaeology 19:215-236.

Gómez Otero, J., J.B. Belardi, R. Tikot y S. Grammer 2000. Dietas y poblaciones humanas en la costa norte del Chubut (Patagonia Argentina). En Desde el País de los Gigantes. Perspectivas 
Arqueológicas en Patagonia, editado por J.B. Berardi, F. Carballo y S. Espinosa, pp. 109-123. Universidad Nacional de la Patagonia Austral, Río Gallegos.

González, L., A. Maldonado, L. Núñez, I. Cartajena, C. Carrasco y P. de Souza 2011. Condiciones paleovegetacionales y asentamientos humanos durante el Formativo análisis de polen del sitio Tulán-85 ( 1.500-400 años cal. a.C.), cuenca del Salar de Atacama. Chungara Revista de Antropología Chilena, en revisión.

Grosjean, M., L. Núñez e I. Cartajena 1997. Mid-Holocene climate and culture change in the Atacama Desert, Northern Chile. Quaternary Research 48:239-246.

Guichón, R., L.A. Borrero, A. Prieto, P. Cárdenas y R. Ticket 2001. Nuevas determinaciones de isótopos estables para Tierra del Fuego. Revista Argentina de Antropología Biológica 3:113-126.

Hedges, R. y L. Reynard 2007. Nitrogen isotopes and the trophic level of humans in archaeology. Journal of Archaeological Science 34:1240-1251.

López, P. 2010 Isótopos Estables en Restos de Camélidos de Quebrada Tulán (II Región-Chile): Uso del Espacio durante el Formativo Temprano (ca. 3.100-2.400 A.P.). Tesis para obtener el grado de Magíster en Antropología, Universidad Católica del Norte-Universidad de Tarapacá, San Pedro de Atacama.

Luebert, F. y R. Pliscoff 2006. Sinopsis Bioclimática y Vegetacional de Chile: Editorial Universitaria, Santiago.

Mengoni Goñalons, L.G. 2007. Camelid management during Inca times in N.W. Argentina: models and archaeozoological indicators. Anthropozoologica 42:129.141.

Newsome, S., D. Phillips, B. Culleton, T. Guilderson y P. Koch 2004. Dietary reconstruction of an early to Middle Holocene human population from Central California coast: Insights from advanced stable isotope mixing models. Journal of Archaeological Science 31:1101-1115.

Novelillo, P., A. Gil, G. Neme y V. Durán 2004. El consumo de maíz en el Holoceno tardío del oeste argentino: isótopos estables y caries. Revista Española de Antropología Americana 34:85-110.

Núñez, L. y C. Santoro 1988. Cazadores de la puna seca y salada del área centro-sur andina (norte de Chile). Estudios Atacameños 9:11-60.

Núñez, L. I. Cartajena, C. Carrasco, P. de Souza. y M. Grosjean 2006. Emergencia de comunidades pastoralistas formativas en el sureste de la Puna de Atacama. Estudios Atacameños 32:93-117.

Núñez, L., M. Grosjean, B. Messerli y H. Schreier 1997. Cambios ambientales holocénicos en la puna de Atacama y sus implicancias paleoclimaticas. Estudios Atacameños 12:31-40.

Núñez, L., V. McRostie e I. Cartajena 2009. Consideraciones sobre la recolección vegetal y la horticultura durante el Formativo Temprano en sureste de la cuenca de Atacama. Darwiniana 47:56-75.
Núñez, M.K. 2000. Movimientos y Voces en Peine. Tesis para obtener el grado de Licenciada en Antropología y al Título de Antropóloga, Escuela de Antropología, Universidad Academia de Humanismo Cristiano, Santiago.

Panarello, H. y J. Fernández 2002. Stable carbon isotope measurenments on hair from wild animal from altiplanic environment of Jujuy. Radiocarbon 44:709-716.

Panarello, H., A. Zangrando, A. Tessone, L. Kozameh, y N. Testa 2006. Análisis comparativo de paleodietas humanas entre la región del canal Beagle y Península Mitre: perspectivas desde los isótopos estables. Magallania 34: 37-46.

Quade, J., J.A. Rech, C. Latorre, J.L. Betancourt, E. Gleeson, M. Kalin 2007. Soils at the hyperarid margin: the isotopic composition of soil carbonate from the Atacama Desert, northern Chile. Geochimica et Cosmochimica Acta 71:3772-3795.

Raedeke, K. y J. Simonetti 1988. Food habitat of Lama guanaco in the Atacama Desert of Northern Chile. Journal of Mammalogy 69:198-201.

Slovak, N., A. Paytan y B. Wiegand 2009. Reconstructing Middle Horizon mobility patterns on the coast of Peru through strontium isotope analysis. Journal of Archaeological Science 36:157-165.

Tieszen, L. y M. Chapman 1992. Carbon and nitrogen isotopic status of the major marine and terrestrial resources in the Atacama desert of northern Chile. Proceedings of the First World Congress on Mummy Studies Volumen 1:409-426. Museo Arqueológico y Etnográfico de Tenerife, Organismo Autónomo de Museos y Centros, Cabildo de Tenerife.

Turner, B., J. Kingston y G. Armelagos 2010. Variation in dietary histories among the immigrants of Machu Picchu: Carbon and Nitrogen isotope evodence. Chungara Revista de Antropología Chilena 42:497-513.

Tykot, R. 2004. Stable isotopes and diet: You are what you eat. En Proceedings of the International School of Physics "Enrico Fermi" Course CLIV, editado por M. Martini, M. Milazzo y M. Piacentini, pp. 433-444. IOS Press, Ámsterdam.

Tykot, R., F. Falabella, M.T. Planella, E. Aspillaga, L. Sanhueza y C. Becker 2009. Stable isotopes and archaeology in central Chile: methodological insights and interpretative problems for dietary reconstruction. International Journal of Ostearchaeology 19:156-170.

Villagrán, C., M. Romo y V. Castro 2003. Etnobotánica del sur de Los Andes de la Primera Región de Chile: un enlace entre las culturas altiplánicas y las quebradas altas del Loa superior. Chungara Revista de Antropología Chilena 35:73-124.

Von den Driesch, A. 1999. A guide to measurement of animal bones from archaeological sites. Peabody Museum Bulletins 1 . Harvard University, Cambridge.

1 Familias Poaceae, Solanaceae, Amaranthaceae, Asteraceae, Euphorbiaceae, Ephedraceae, Portulacaceae y Fabaceae. 
